\title{
Transthyretin-A Key Gene Involved in Regulating Learning and Memory in Brain, and Providing Neuroprotection in Alzheimer Disease via Neuronal Synthesis of Transthyretin Protein
}

\author{
Javed Iqbal \\ College of Life Science, Shaanxi Normal University, Xi'an, China \\ Email: javed@snnu.edu.cn
}

How to cite this paper: Iqbal, J. (2018) Transthyretin-A Key Gene Involved in Regulating Learning and Memory in Brain, and Providing Neuroprotection in Alzheimer Disease via Neuronal Synthesis of Transthyretin Protein. Journal of Behavioral and Brain Science, 8, 77-92.

https://doi.org/10.4236/jbbs.2018.82005

Received: December 29, 2017

Accepted: February 10, 2018

Published: February 13, 2018

Copyright $\odot 2018$ by author and Scientific Research Publishing Inc. This work is licensed under the Creative Commons Attribution International License (CC BY 4.0).

http://creativecommons.org/licenses/by/4.0/

\begin{abstract}
Transthyretin (TTR), a carrier protein present in the liver and choroid plexus of the brain, has been shown to be responsible for binding thyroid hormone thyroxin (T4) and retinol in plasma and cerebrospinal fluid (CSF). TTR aids in sequestering of beta-amyloid peptides $\mathrm{A} \beta$ deposition, and protects the brain from trauma, ischemic stroke and Alzheimer disease (AD). Accordingly, hippocampal gene expression of TTR plays a significant role in learning and memory as well as in simulation of spatial memory tasks. TTR via interacting with transcription factor CREB regulates this process and decreased expression leads to memory deficits. By different signaling pathways, like MAPK, AKT, and ERK via Src, TTR provides tropical support through megalin receptor by promoting neurite outgrowth and protecting the neurons from traumatic brain injury. TTR is also responsible for the transient rise in intracellular $\mathrm{Ca}^{2+}$ via NMDA receptor, playing a dominant role under excitotoxic conditions. In this review, we tried to shed light on how TTR is involved in maintaining normal cognitive processes, its role in learning and memory, under memory deficit conditions; by which mechanisms it promotes neurite outgrowth; and how it protects the brain from Alzheimer disease (AD).
\end{abstract}

\section{Keywords}

Learning and Memory, TTR-Transthyretin, AD-Alzheimer Disease, CSF-Cerebrospinal Fluid, MAPK-Mitogen-Activated Protein Kinases, CREB-CAMP Response Element Binding Protein, ERK-Extracellular Receptor Kinases, $\mathrm{A} \beta$-Amyloid Beta, LTP-Long-Term Potentiation, LTD-Long-Term Depression 


\section{Introduction}

Transthyretin (TTR), a carrier protein for thyroxine and retinol present in the plasma and cerebrospinal fluid (CSF), has been shown to sequester the amyloid beta-peptide deposition in the brain [1] [2]. The transthyretin gene is mainly expressed in the liver and choroid plexus of the brain. Transthyretin plays a very important role in binding and distribution of thyroid hormones in the body and brain to protect it during neurological strokes [3] [4]. The choroid plexus of the brain secretes TTR at a rate 13 times greater than liver [5]. The total TTR proteins contents account for at least $20 \%$ of all synthesized by the choroid plexus in the rat brain. It has been suggested that uni-directional secretion of TTR proteins to the CSF plays a key role in the transfer of thyroxine (T4) from the blood to the CSF, as TTR may drive thyroxin hormones (TH) across the blood-CSF barrier [6]. TTR is a major distributor of T4 in the CSF and its distribution from CSF to the brain is dependent on TTR based receptor-mediated endocytosis process [7] [8] [9]. Although, TTR possesses the higher affinity for thyroxine binding proteins (TGB) than T4, it may only involve in the peripheral circulation. TTR-T4 binding can be seen in the CSF promoting T4 transport from blood to brain [10]. TTR is also indirectly implicated in the transport of vitamin A through interaction with retinol-binding protein (RBP) [11].

Besides its role as a carrier protein, TTR has been involved in the sequestering of $\beta$-amyloid peptide $(\mathrm{A} \beta)$ in the brain, protecting the brain from neurotoxic accumulations called amyloid plaques. However, it is unclear whether TTR affects the clearance of $\beta$-amyloid from the CSF [10]. In vitro studies showed that TTR via its TGB interacts with $\mathrm{A} \beta$ residues, preventing its toxicity in the brain cells [12] [13] [14] [15]. TTR is suggested to be a protease molecule with apolipoprotein structure A-I (apoA-I) and $\mathrm{A} \beta$ as substrates. TTR cleaves $\mathrm{A} \beta$ at multiple positions to degrade aggregated forms of $\mathrm{A} \beta$. In vivo studies suggested that TTR can transport $\mathrm{A} \beta$ from the brain to blood but not into the brain [16]. TTR crosses the monolayer of cells only in the brain-to-blood direction. TTR-mediated A $\beta$ clearance is through LRP1, as lower receptor expression was found in brains and livers of TTR knockdown mice and in cells incubated without TTR [16] [17]. The activated TTR may contribute not only to the upholding of $A \beta$ levels within a normal range but also to the degradation of deposited $\mathrm{A} \beta$ in the case of imbalance and diseases condition [18].

This review addresses some of the key questions like

1) TTR-A neuroprotective and neurite growth protein.

2) TTR-important contributor in learning and memory.

3) Special role with respect to Alzheimer Disease (AD).

\section{Transthyretin Promotes Neurite Outgrowth in Hippocampal Neurons and Provides Neuroprotection}

TTR protein has the ability to enhance neurite outgrowth in vitro, plays apivotal role in nerve regeneration process and provides neuroprotection in the peri- 
pheral and central nervous system [19] [20] [21]. In vivo and in-vitro studies showed that, in the absence of TTR, both hippocampal neurite outgrowth and nerve regeneration were impaired [20] [22]. TTR significantly boosted neurite outgrowth via megalin receptor in hippocampal neurons, both in number and length [23] [24]. Neuronal cell lines exposed to TTR KO serum displayed 25\% decrease in neurite number per cell and in the length of the longest neurite in cell culture, when compared to cells grown with WT culture. To ascertain whether the absence of TTR protein was directly liable for this decreased in neurite number and size, TTR KO serum supplemented with wild type TTR was able to totally rescue the phenotype observed in the absence of the protein [25]. Several studies pointed that it can be a good predictor for stroke patients exhibiting decreased serum TTR and young women, owing to the neuroprotective action of steroids that are up-regulating TTR [26] [27] [28] [29]. Familial amyloid polyneuropathy (FAP), a neurodegenerative disorder is caused by TTR deposition in the peripheral nervous system (PNS). The reason for TTR deposition in the nerve leading to FAP is still unknown. When TTR was delivered in TTR knock-out (KO) mice, the sciatic nerve regeneration phenotype was rescued.

TTR binds several different proteins including megalin (LRP-2), RAGE (receptor for advanced glycation end products) and IGF-IR (insulin-like growth factor 1 receptor) [30] [31] [32] [33] [34] to activate the downstream signaling pathways. These signaling pathways activated by TTR in the CNS lead to neuroprotection under physiological and pathological conditions involving TTR dependent activation of mitogen-activated protein kinase (MAPK), extracellular receptor kinases (ERK1/2) and Akt through Src, leading to the phosphorylation of transcription factor CREB (Figure 1) [35] [36]. In addition, TTR promotes a transient rise in intracellular calcium through anN-methyl-D-aspartate receptor (also known as the NMDA receptor), in a Src/megalin-dependent manner [36]. The megalin molecule possesses Src homology domain (SH3) constitutes a crucial point of convergence for signaling pathways. This bridging molecule Src could be responsible for the increase in calcium level inside neurons via activating NMDA receptor because Src regulates NMDA receptor activity [35] [37] [38].

The second pathway through which TTR stimulation rescues cell death and neurite loss is by activating $\mathrm{Bcl} 2$ protein family members in megalin-dependent manners. TTR stimulation under excitotoxic conditions rescued TTR KO hippocampal neurons in a megalin-dependent manner, which were more sensitive to excitotoxic degeneration than WT neurons [39]. In this process, TTR activates CREB, contributing to changes in the balance between $\mathrm{Bcl} 2$ protein family members, toward anti-apoptotic proteins (Bcl2/BclXL compared to Bax protein) [23] [40] [41] [42]. TTR triggered by its interaction with a well-known neuroprotective megalin-dependent signaling pathways promotes a robust neurite outgrowth response in hippocampal neurons by the upregulation of intracellular calcium and MAPK pathways (Figure 1). Megalin was identified 


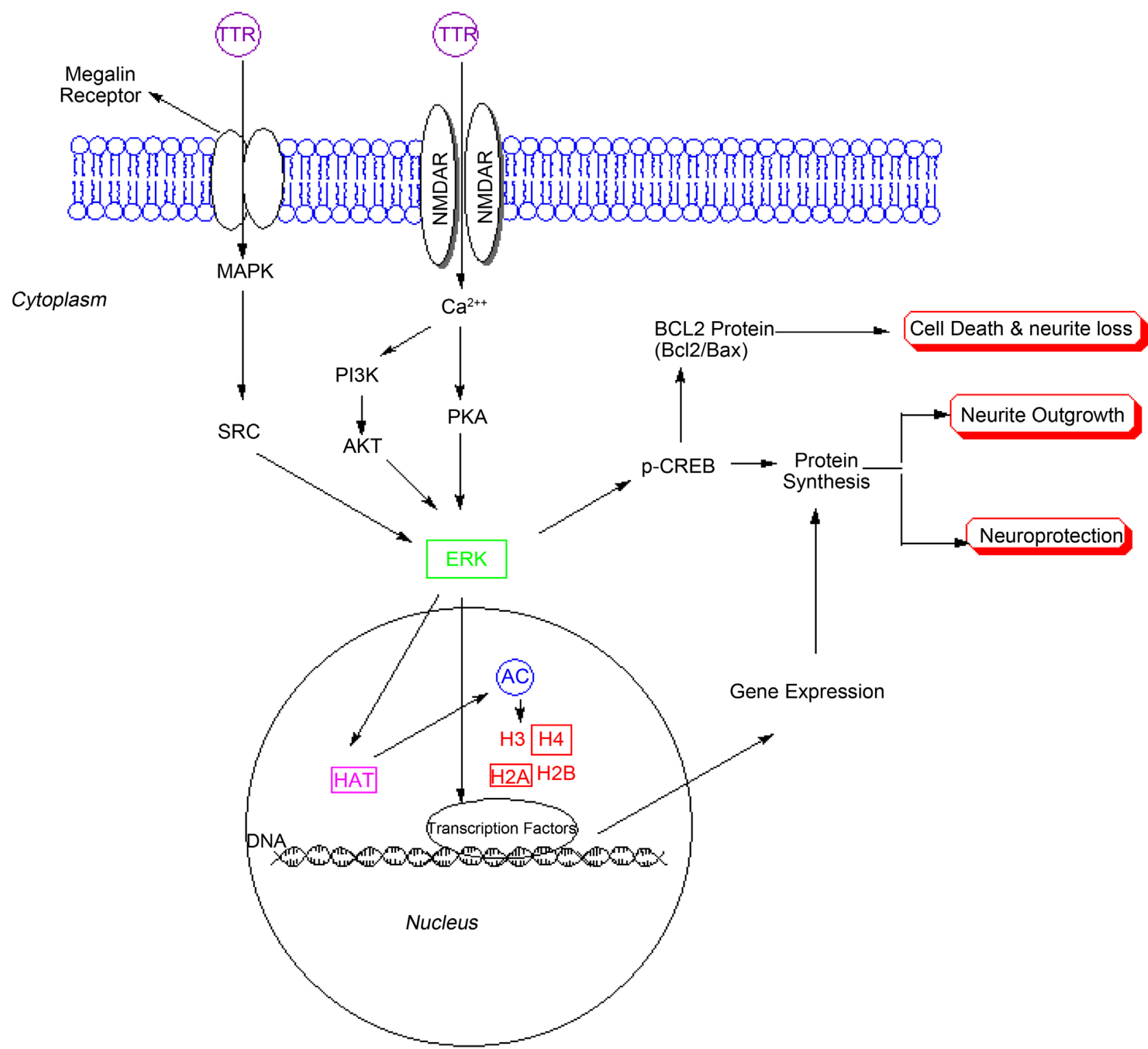

Figure 1. TTR via different signaling pathways responsible for phosphorylation and increase of intracellular calcium level lead to promote neurite outgrowth and provides neuroprotection with apoptotic gene under excitotoxic conditions.

as the receptor involved in the transduction of TTR neuroprotection via megalin in hippocampal neuronal cell culture [23]. TTR interaction with megalin was found to be important determinant for neuronal survival and neurite preservation both in excitotoxic conditions and in a mouse model of permanent middle cerebral artery occlusion (pMCAO). As megalin was necessary for the neuroprotective action of TTR, megalin mRNA levels was quantified after glutamate stimulation in both TTR KO and WT cultures. Megalin mRNA was found to be significantly upregulated in WT cultures in opposition to TTR KO cultures indicating that megalin is required for proper functioning of TTR. TTR up-regulates intracellular calcium and takes Src/ErK/Akt/CREB pathway in a megalin-dependent manner both in vitro and in vivo. Moreover, clathrin-dependent mega- 
lin-mediated TTR internalization is required for TTR neurogenic activity in dorsal root ganglia neurons. Supporting this notion, it was demonstrated that megalin's action as an enhancer of nerve regeneration is dependent on TTR expression and decrease levels of megalin lead to slow down the nerve regeneration process [20] [43]. Keeping in view the above discussions, TTR might be regarded as a neurotrophic factor, both in neurite outgrowth and neuroprotection under physiological conditions.

\section{Role in the Maintenance of Learning and Memory Capacities of Brain}

In this section, we ascribed the questions like how TTR regulate learning and memory? Which mechanism is more critical in maintaining it? Does TTR also contribute to play its different role during aging process? To shed light on these aspects, we have tried through a reductionist approach.

Learning and memory is a complex process accompanied by molecular modifications and is driven by hippocampus genes [44]. To elucidate the molecular mechanisms responsible for stable forms of long-term memory consolidation require the induction of a cascade of genes responsible for producing and maintaining structural changes associated with memory formation. Despite all these molecular changes that are enhancing memory, little is known about molecular events most relevant to recover learning and memory deficit [45] [46]. Emerging evidence on protein interaction networks that monitor and respond to the normal memory enhancing process suggested that TTR is involved in neurodegenerative disorders like Alzheimer disease [47].

The hippocampus, a structure essential for the formation and retrieval of spatial and reference memory, is necessary for long-term memory consolidation or storage [48] [49] [50]. Genome-wide study investigated genes differentially expressed between aged memory-impaired (AI) and aged memory-unimpaired (AU) rats [51] [52] [53] [54] [55]. Long-term memory formation depends on two distinct parameters, 1) gene expression and 2) protein synthesis [55]. TTR is one of the most markedly affected genes between the two groups: aged memory-impaired (AI) and aged memory-unimpaired (AU) rats [56]. Studies showed that TTR is a critical component of the retinoid pathway for the maintenance of memory capacities during aging [56] [57] [58] [59] [60]. The upregulation of TTR is essential to prevent from memory deficits. During AI and AU conditions, TTR gene expression was affected most markedly. Decrease TTR gene expression may considerably alter the bioavailability of retinoic acid (RA) in response to the body's needs because TTR delivers retinol to the target tissue as well [57]. This could be attributed partially to diminish the activity of C/EBP (a transcription factor regulating both TTR and NQO2 expression) immediate-early gene cascade initiated by CREB since C/EBP protein levels were decreased in AI animals [56] [61] [62]. Genetic, behavioral studies and molecular evidence supported the notion that TTR is involved in the maintenance of normal cognitive 
processes during aging by acting on the retinoid signaling pathway [3] [56]. TTR and RA deficiency in the hippocampus may decrease memory performance by impairing synaptic plasticity [58], long-term potentiation (LTP) and long-term depression (LTD) [63] [64].

TTR influence learning and spatial memory in a robust way. Studies carried out using TTR knockdown mice demonstrated that it regulate the cognitive process and protect the brain from traumatic injury [3]. Hippocampus-dependent memory impairment is associated with synaptic dysfunction/alterations that occur during normal aging [65] [66]. Several lines of studies pointed that TTR accelerates the cognitive decline resulting from aging. Impairment resulting from TTR knockdown gene also temporary proceeds to functional deficits in processes mediated by prefrontal cortex [67]-[72]. TTR-null mice showed increase neuropeptide $Y$ levels in the hippocampus [73]. Over-expression of neuropeptide $\mathrm{Y}$ in the hippocampus of transgenic rats induces spatial memory impairment, which could also be interconnected to the cognitive phenotype in TTR knockdown mice [74].

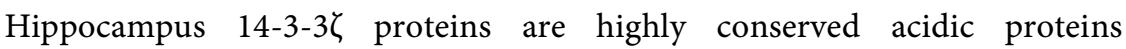
representing $1 \%$ of the total amount of brain proteins and very important in many cellular processes, being their absence related to memory deficits and learning [75]. In neurons, this protein exists in the cytoplasm, in mitochondria, and also in the nucleus [76] [77]. Several studies revealed that TTR regulates 14-3-3 $\zeta$ protein levels in the hippocampus [73] [75]. TTR knockdown mice had decreased levels of 14-3-3 $\zeta$ protein in the hippocampus and this decreased level impact on learning and memory [78]. The cognitive decline with decreased expression is strongly connected with synaptic activity-dependent proteins [78]. Western blot and immunohistochemistry analysis described the increase degra-

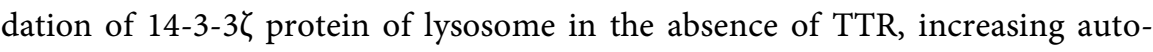
phagy and memory deficits process [38] [79] [80] [81] [82]. In conclusion, the absence of TTR decreases 14-3-3 $\zeta$ protein levels in the hippocampus which in turn attributable to increased degradation in the lysosomal compartments, probably by the reduced levels of $14-3-3 \zeta$ protein [83] [84].

\section{TTR Role in Alzheimer Disease}

Alzheimer disease is a neurodegenerative disorder primary characterized by the extracellular deposition of $\mathrm{A} \beta$ and the formation of neurofibrillary tangles in the brain [85] [86] [87]. In $\mathrm{AD}$ patients, two different mechanisms are involved, overproduction versus accumulation of soluble $\mathrm{A} \beta$ [88]. In both cases, sequestering of $\mathrm{A} \beta$ contribute to its clearance from the brain and protect against accumulation or susceptibility to diseases. The TTR plays pivotal role during this process by activating downstream signaling events leading to $\mathrm{A} \beta$ sequestering. The hallmark of TTR is its role in Alzheimer's disease, protecting the brain from accumulating neurotoxic amyloidplaques. TTR suppressed $\mathrm{A} \beta$ and prevent its toxicity [89] [90]. Studies showed that TTR sequestered $A \beta$ in the CSF and 
moved $\mathrm{A} \beta$ to the choroid plexus (CP) for enzymatic degradation and removal from the brain [18]. In this context, TTR-A $\beta$ interaction seems to have a neuroprotective effect in AD patients [91] [92] [93]. Brain TTR exhibits asymmetrical expression pattern and was confirmed in normal and transgenic mouse model in AD condition [94]. The levels of TTR in the brain of AD patients are correlated negatively with the abundance and accumulations of amyloid plaques. In response to elevated $\mathrm{A} \beta$ levels, the TTR is up regulated in transgenic mice overexpressing mutant amyloid precursor protein [95] [96].

Patients suffering from AD showed significant reduction of TTR concentration in the CSF compared to age-matched healthy control subjects suggesting that $\mathrm{AD}$ patients expressed decrease TTR level with an enhanced level of $\mathrm{A} \beta$ in the brain ( $p=0.004)$ [97] [98] [99] [100]. Gene expression of TTR can be induced in response to overproduction of $\mathrm{A} \beta$ but this seems to be in contrast to the observations that the concentration of TTR is lower in most cases, if not all, AD patients than in aged-match control groups [96] [101] [102]. In situ hybridization analysis identified very low level of TTR mRNA transcripts in the hippocampus while the majority was localized in the choroid plexus of the brain [103]. Results from the experiment using C57BL/6JNarl mice and Tg2576 mice showed that not only the level but also the hemispheric symmetry of TTR expression in the mouse brain was significantly altered in the AD mouse model Tg2576 [94]. Interestingly, in this study, the levels of TTR transcripts in the hemispheres of the adult females, aged females, and aged males were all higher than the adult males. Furthermore, this asymmetrical distribution of TTR in the brain hemispheres might have an important link with the pathogenesis of the $\mathrm{AD}$ [104] [105].

The implication of TTR as a neuroprotective agent in AD was further validated by two well-characterized models of AD: the APP-V717I and the Tg2576 (K670N, M671L) transgenic mice, described to display cognitive impairment and amyloid plaques [68] [107] [108]. By immunohistochemistry analysis, TTR immunoreactivity was restricted to the choroid plexus of the brain. These two validated models of AD clearly showed that TTR expression can be seen in choroid plexus and mutation to TTR can lead to disease pathology [68].

Oxidative stress and inflammation also contribute to $\mathrm{AD}$. Multiple genes and molecular mechanisms have been implicated in this process. Significantly altered gene expression was found in AD-affected Choroid Plexus Epithelium (CPE) [109]. Specific cellular changes were observed due to increased oxidative stress. Most likely, $\mathrm{CPE}$ sink action may be impaired in $\mathrm{AD}$ patients due to down regulation of CLDN5 gene. $\mathrm{A} \beta \mathrm{PP}$ processing pathways are responsible for $\mathrm{AD}$ condition (Figure 2) [106]. Reduced TTR levels in CSF have also been reported in $\mathrm{AD}$ patients with depression. The inconsistency among the finding has made it an unsuitable CSF marker for AD patients and made it more complicated to understand its role in $\mathrm{AD}$ pathogenesis. The third way, the TTR affect AD model depends upon its function as an RBP binding protein. Increased TTR indirectly 


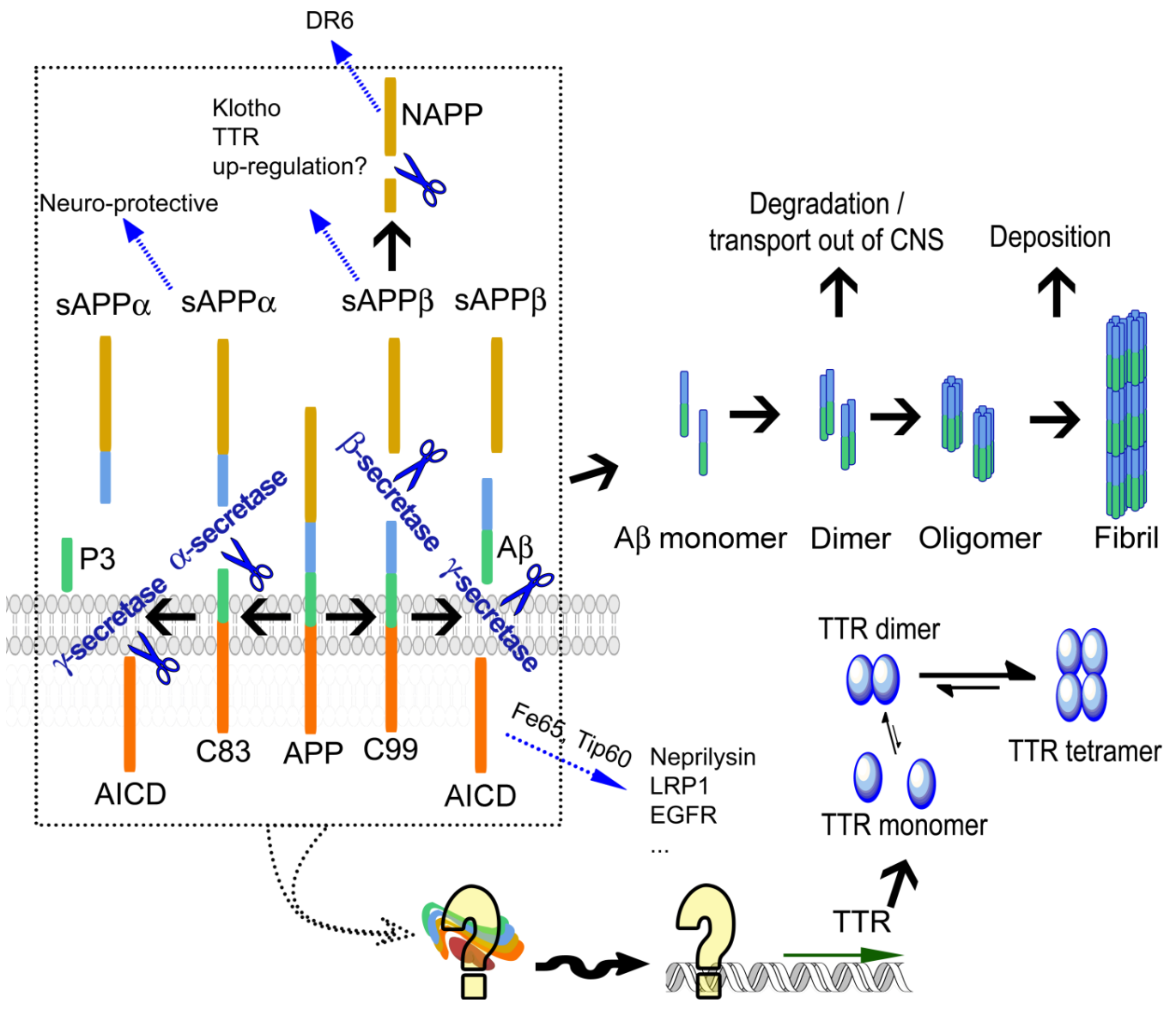

Figure 2. Amyloid precursor protein (APP) processing pathway during Alzheimer Disease. AICD, AbPP intracellular domain; C83 (C99), carboxy-terminal fragments C83 (C99); DR6, death receptor 6; LRP, lipoprotein receptor-related protein; sAPP, secreted AbPP fragment; TTR, transthyretin. Degradation, see "Clearance of Ab" of the text for details. Involvement of TTR regulation: it has been suggested that APP or its fragments upregulate TTR [106].

increases the amount of available retinoic acid in the brain, thus enhancing neuronal maintenance. Similarly, the accelerating effect could also depend on a relative lack of retinoid in the CNS which amplifies $\mathrm{A} \beta$. RBP inhibitors have been shown to compromise neuronal function in aged rodents whereas retinoic acid has been found to enhance performance [56] [110].

Despite the stable role of TTR in the CNS and AD, it is far to understood the specific and pivotal role of TTR's site of synthesis in AD. Altered TTR gene expression in the choroid plexus and the TTR levels in CSF consequently could impact on AD pathology and disease prognosis. Further studies are needed to explore the main domain of TTR in AD so that it can be recovered in a more sophisticated way. The scenario like that could certainly account for the findings in the overexpression models of $\mathrm{AD}$, whether it applies to sporadic disease is a subject matter of speculation and further investigation. Any such studies could not ignore the possible role of TTR since cortical and hippocampal neurons from human $\mathrm{AD}$ and mouse $\mathrm{AD}$ model brains seem to increase its production [106]. 


\section{Conclusion}

TTR plays a significant novel role in sequestering of beta-amyloid peptides deposition in the brain, promotes neurite extension and provides neural protection in Alzheimer disease. TTR plays an important role in nerve regeneration process supporting a pivotal role for TTR in neurobiology. It regulates learning and memory process in the hippocampus and decreased expression of TTR leads to memory deficits in brain. It has been widely studied related to AD but further investigations are needed to explore its role in different brain-related diseases.

\section{References}

[1] Ribeiro, C.A., et al. (2014) Transthyretin Stabilization by Iododiflunisal Promotes Amyloid-Beta Peptide Clearance, Decreases Its Deposition, and Ameliorates Cognitive Deficits in an Alzheimer's Disease Mouse Model. Journal of Alzheimer's Disease, 39, 357-370

[2] Palha, J.A. (2002) Transthyretin as a Thyroid Hormone Carrier: Function Revisited. Clinical Chemistry and Laboratory Medicine, 40, 1292-1300. https://doi.org/10.1515/CCLM.2002.223

[3] Sousa, J.C., et al. (2007) Transthyretin Influences Spatial Reference Memory. Neurobiology of Learning and Memory, 88, 381-385.

https://doi.org/10.1016/j.nlm.2007.07.006

[4] Bauer, M., Heinz, A. and Whybrow, P.C. (2002) Thyroid Hormones, Serotonin and Mood: Of Synergy and Significance in the Adult Brain. Molecular Psychiatry, 7, 140-156. https://doi.org/10.1038/sj.mp.4000963

[5] Dickson, P.W. and Schreiber, G. (1986) High Levels of Messenger RNA for Transthyretin (Prealbumin) in Human Choroid Plexus. Neuroscience Letters, 66, 311-315. https://doi.org/10.1016/0304-3940(86)90037-6

[6] Southwell, B.R., et al. (1993) Thyroxine Transport to the Brain: Role of Protein Synthesis by the Choroid Plexus. Endocrinology, 133, 2116-2126. https://doi.org/10.1210/endo.133.5.8404661

[7] Hu, S., Loo, J.A. and Wong, D.T. (2006) Human Body Fluid Proteome Analysis. Proteomics, 6, 6326-6353. https://doi.org/10.1002/pmic.200600284

[8] Kassem, N.A., et al. (2006) Role of Transthyretin in Thyroxine Transfer from Cerebrospinal Fluid to Brain and Choroid Plexus. American Journal of Physiology. Regulatory, Integrative and Comparative Physiology, 291, R1310-R1315. ps://doi.org/10.1152/ajpregu.00789.2005

[9] Chen, R.L., Kassem, N.A. and Preston, J.E. (2006) Dose-Dependent Transthyretin Inhibition of T4 Uptake from Cerebrospinal Fluid in Sheep. Neuroscience Letters, 396, 7-11. https://doi.org/10.1016/j.neulet.2005.11.003

[10] Chen, R., Chen, C.P. and Preston, J.E. (2016) Effects of Transthyretin on Thyroxine and Beta-Amyloid Removal from Cerebrospinal Fluid in Mice. Clinical and Experimental Pharmacology \& Physiology, 43, 844-850. https://doi.org/10.1111/1440-1681.12598

[11] Suzuyama, K., et al. (2004) Combined Proteomic Approach with SELDI-TOF-MS and Peptide Mass Fingerprinting Identified the Rapid Increase of Monomeric Transthyretin in Rat Cerebrospinal Fluid after Transient Focal Cerebral Ischemia. Molecular Brain Research, 129, 44-53.

https://doi.org/10.1016/j.molbrainres.2004.06.021 
[12] Schwarzman, A.L., et al. (1994) Transthyretin Sequesters Amyloid Beta Protein and Prevents Amyloid Formation. Proceedings of the National Academy of Sciences of the United States of America, 91, 8368-8372.

[13] Li, X., et al. (2013) Mechanisms of Transthyretin Inhibition of Beta-Amyloid Aggregation In Vitro. Journal of Neuroscience, 33, 19423-19433. https://doi.org/10.1523/JNEUROSCI.2561-13.2013

[14] Yang, D.T., et al. (2013) Transthyretin as Both a Sensor and a Scavenger of Beta-Amyloid Oligomers. Biochemistry, 52, 2849-2861. https://doi.org/10.1021/bi4001613

[15] Schultz, K., Traskman-Bendz, L. and Petersen, A. (2008) Transthyretin in Cerebrospinal Fluid from Suicide Attempters. Journal of Affective Disorders, 109, 205-208. https://doi.org/10.1016/j.jad.2007.11.007

[16] Alemi, M., et al. (2016) Transthyretin Participates in Beta-Amyloid Transport from the Brain to the Liver-Involvement of the Low-Density Lipoprotein Receptor-Related Protein 1? Scientific Reports, 6, Article Number: 20164. https://doi.org/10.1038/srep20164

[17] Oliveira, S.M., et al. (2011) Gender-Dependent Transthyretin Modulation of Brain Amyloid-Beta Levels: Evidence from a Mouse Model of Alzheimer's Disease. Journal of Alzheimer's Disease, 27, 429-439.

[18] Costa, R., et al. (2008) Transthyretin Protects against A-Beta Peptide Toxicity by Proteolytic Cleavage of the Peptide: A Mechanism Sensitive to the Kunitz Protease Inhibitor. PLOS ONE, 3, e2899. https://doi.org/10.1371/journal.pone.0002899

[19] Alshehri, B., et al. (2015) The Diversity of Mechanisms Influenced by Transthyretin in Neurobiology: Development, Disease and Endocrine Disruption. Journal of Neuroendocrinology, 27, 303-323. https://doi.org/10.1111/jne.12271

[20] Fleming, C.E., et al. (2009) Transthyretin Internalization by Sensory Neurons Is Megalin Mediated and Necessary for Its Neuritogenic Activity. Journal of Neuroscience, 29, 3220-3232. https://doi.org/10.1523/JNEUROSCI.6012-08.2009

[21] Goncalves, N.P., Teixeira-Coelho, M. and Saraiva, M.J. (2015) Protective Role of Anakinra against Transthyretin-Mediated Axonal Loss and Cell Death in a Mouse Model of Familial Amyloidotic Polyneuropathy. Journal of Neuropathology \& EXperimental Neurology, 74, 203-217. https://doi.org/10.1097/NEN.0000000000000164

[22] Doggui, S., et al. (2010) Possible Involvement of Transthyretin in Hippocampal Beta-Amyloid Burden and Learning Behaviors in a Mouse Model of Alzheimer's Disease (TgCRND8). Neurodegenerative Diseases, 7, 88-95. https://doi.org/10.1159/000285513

[23] Gomes, J.R., et al. (2016) Transthyretin Provides Trophic Support via Megalin by Promoting Neurite Outgrowth and Neuroprotection in Cerebral Ischemia. Cell Death and Differentiation, 23, 1749-1764. https://doi.org/10.1038/cdd.2016.64

[24] Alvira-Botero, X., et al. (2010) Megalin Interacts with APP and the Intracellular Adapter Protein FE65 in Neurons. Molecular and Cellular Neuroscience, 45, 306-315. https://doi.org/10.1016/j.mcn.2010.07.005

[25] Fleming, C.E., et al. (2009) Chapter 17: Transthyretin: An Enhancer of Nerve Regeneration. International Review of Neurobiology, 87, 337-346. https://doi.org/10.1016/S0074-7742(09)87017-7

[26] Santos, S.D., et al. (2010) CSF Transthyretin Neuroprotection in a Mouse Model of Brain Ischemia. Journal of Neurochemistry, 115, 1434-1444.

https://doi.org/10.1111/j.1471-4159.2010.07047.x 
[27] Gao, C., et al. (2011) Serum Prealbumin (Transthyretin) Predict Good Outcome in Young Patients with Cerebral Infarction. Clinical and Experimental Medicine, 11, 49-54. https://doi.org/10.1007/s10238-010-0103-8

[28] Quintela, T., et al. (2009) 17Beta-Estradiol Induces Transthyretin Expression in Murine Choroid Plexus via an Oestrogen Receptor Dependent Pathway. Cellular and Molecular Neurobiology, 29, 475-483.

https://doi.org/10.1007/s10571-008-9339-1

[29] Garzuly, F., et al. (1996) Familial Meningocerebrovascular Amyloidosis, Hungarian Type, with Mutant Transthyretin (TTR Asp18Gly). Neurology, 47, 1562-1567. https://doi.org/10.1212/WNL.47.6.1562

[30] Sousa, M.M. and Saraiva, M.J. (2001) Internalization of Transthyretin. Evidence of a Novel yet Unidentified Receptor-Associated Protein (RAP)-Sensitive Receptor. The Journal of Biological Chemistry, 276, 14420-14425. https://doi.org/10.1074/jbc.M010869200

[31] Wang, X., et al. (2010) Caenorhabditis elegans Transthyretin-Like Protein TTR-52 Mediates Recognition of Apoptotic Cells by the CED-1 Phagocyte Receptor. Nature Cell Biology, 12, 655-664. https://doi.org/10.1038/ncb2068

[32] Sousa, M.M., et al. (2000) Evidence for the Role of Megalin in Renal Uptake of Transthyretin. The Journal of Biological Chemistry, 275, 38176-38181. https://doi.org/10.1074/jbc.M002886200

[33] Sousa, M.M., et al. (2000) Interaction of the Receptor for Advanced Glycation End Products (RAGE) with Transthyretin Triggers Nuclear Transcription Factor kB (NF-kB) Activation. Laboratory Investigation, 80, 1101-1110. https://doi.org/10.1038/labinvest.3780116

[34] Vieira, M., Gomes, J.R. and Saraiva, M.J. (2015) Transthyretin Induces Insulin-Like Growth Factor I Nuclear Translocation Regulating Its Levels in the Hippocampus. Molecular Neurobiology, 51, 1468-1479. https://doi.org/10.1007/s12035-014-8824-4

[35] Marzolo, M.P. and Farfan, P. (2011) New Insights into the Roles of Megalin/LRP2 and the Regulation of Its Functional Expression. Biological Research, 44, 89-105. https://doi.org/10.4067/S0716-97602011000100012

[36] Mantuano, E., et al. (2008) Molecular Dissection of the Human Alpha ${ }_{2}$-Macroglobulin Subunit Reveals Domains with Antagonistic Activities in Cell Signaling. The Journal of Biological Chemistry, 283, 19904-19911.

https://doi.org/10.1074/jbc.M801762200

[37] Salter, M.W. and Kalia, L.V. (2004) Src Kinases: A Hub for NMDA Receptor Regulation. Nature Reviews Neuroscience, 5, 317-328. https://doi.org/10.1038/nrn1368

[38] Nunes, A.F., et al. (2009) Transthyretin Knockout Mice Display Decreased Susceptibility to AMPA-Induced Neurodegeneration. Neurochemistry International, 55, 454-457. https://doi.org/10.1016/j.neuint.2009.07.001

[39] Lai, T.W., Zhang, S. and Wang, Y.T. (2014) Excitotoxicity and Stroke: Identifying Novel Targets for Neuroprotection. Progress in Neurobiology, 115, 157-188. https://doi.org/10.1016/j.pneurobio.2013.11.006

[40] Hill, M.D., et al. (2012) Safety and Efficacy of NA-1 in Patients with Iatrogenic Stroke after Endovascular Aneurysm Repair (ENACT): A Phase 2, Randomised, Double-Blind, Placebo-Controlled Trial. The Lancet Neurology, 11, 942-950. https://doi.org/10.1016/S1474-4422(12)70225-9

[41] Szydlowska, K. and Tymianski, M. (2010) Calcium, Ischemia and Excitotoxicity. Cell Calcium, 47, 122-129. https://doi.org/10.1016/j.ceca.2010.01.003 
[42] Neumann, B., et al. (2015) EFF-1-Mediated Regenerative Axonal Fusion Requires Components of the Apoptotic Pathway. Nature, 517, 219-222. https://doi.org/10.1038/nature14102

[43] Fleming, C.E., Saraiva, M.J. and Sousa, M.M. (2007) Transthyretin Enhances Nerve Regeneration. Journal of Neurochemistry, 103, 831-839. https://doi.org/10.1111/j.1471-4159.2007.04828.x

[44] Mattson, M.P. and Magnus, T. (2006) Ageing and Neuronal Vulnerability. Nature Reviews Neuroscience, 7, 278-294. https://doi.org/10.1038/nrn1886

[45] Kandel, E.R. (2001) The Molecular Biology of Memory Storage: A Dialogue between Genes and Synapses. Science, 294 1030-1038. https://doi.org/10.1126/science.1067020

[46] Silva, A.J. (2003) Molecular and Cellular Cognitive Studies of the Role of Synaptic Plasticity in Memory. Journal of Neurobiology, 54, 224-237.

[47] Gonzalez-Marrero, I., et al. (2015) Choroid Plexus Dysfunction Impairs Beta-Amyloid Clearance in a Triple Transgenic Mouse Model of Alzheimer's Disease. Frontiers in Cellular Neuroscience, 9, 17. https://doi.org/10.3389/fncel.2015.00017

[48] Bach, M.E., et al. (1999) Age-Related Defects in Spatial Memory Are Correlated with Defects in the Late Phase of Hippocampal Long-Term Potentiation In Vitro and Are Attenuated by Drugs That Enhance the cAMP Signaling Pathway. Proceedings of the National Academy of Sciences of the United States of America, 96, 5280-5285. https://doi.org/10.1073/pnas.96.9.5280

[49] Tombaugh, G.C., et al. (2002) Theta-Frequency Synaptic Potentiation in CA1 In Vitro Distinguishes Cognitively Impaired from Unimpaired Aged Fischer 344 Rats. The Journal of Neuroscience, 22, 9932-9940.

[50] Hollup, S.A., et al. (2001) Impaired Recognition of the Goal Location during Spatial Navigation in Rats with Hippocampal Lesions. The Journal of Neuroscience, 21, 4505-4513.

[51] Blalock, E.M., et al. (2003) Gene Microarrays in Hippocampal Aging: Statistical Profiling Identifies Novel Processes Correlated with Cognitive Impairment. The Journal of Neuroscience, 23, 3807-3819.

[52] Lu, T., et al. (2004) Gene Regulation and DNA Damage in the Ageing Human Brain. Nature, 429, 883-891. https://doi.org/10.1038/nature02661

[53] Verbitsky, M., et al. (2004) Altered Hippocampal Transcript Profile Accompanies an Age-Related Spatial Memory Deficit in Mice. Learning \& Memory, 11, 253-260. https://doi.org/10.1101/lm.68204

[54] Stemmelin, J., et al. (2000) Immunohistochemical and Neurochemical Correlates of Learning Deficits in Aged Rats. Neuroscience, 96, 275-289. https://doi.org/10.1016/S0306-4522(99)00561-8

[55] Igaz, L.M., et al. (2002) Two Time Periods of Hippocampal mRNA Synthesis Are Required for Memory Consolidation of Fear-Motivated Learning. The Journal of Neuroscience, 22, 6781-6789.

[56] Brouillette, J. and Quirion, R. (2008) Transthyretin: A Key Gene Involved in the Maintenance of Memory Capacities during Aging. Neurobiology of Aging, 29, 1721-1732. https://doi.org/10.1016/j.neurobiolaging.2007.04.007

[57] Monaco, H.L. (2000) The Transthyretin-Retinol-Binding Protein Complex. Biochimica et Biophysica Acta, 1482, 65-72. https://doi.org/10.1016/S0167-4838(00)00140-0 
[58] Misner, D.L., et al. (2001) Vitamin A Deprivation Results in Reversible Loss of Hippocampal Long-Term Synaptic Plasticity. Proceedings of the National Academy of Sciences of the United States of America, 98, 11714-11719. https://doi.org/10.1073/pnas.191369798

[59] Etchamendy, N., et al. (2001) Alleviation of a Selective Age-Related Relational Memory Deficit in Mice by Pharmacologically Induced Normalization of Brain Retinoid Signaling. The Journal of Neuroscience, 21, 6423-6429.

[60] Cocco, S., et al. (2002) Vitamin A Deficiency Produces Spatial Learning and Memory Impairment in Rats. Neuroscience, 115, 475-482. https://doi.org/10.1016/S0306-4522(02)00423-2

[61] Chen, A., et al. (2003) Inducible Enhancement of Memory Storage and Synaptic Plasticity in Transgenic Mice Expressing an Inhibitor of ATF4 (CREB-2) and C/EBP Proteins. Neuron, 39, 655-669. https://doi.org/10.1016/S0896-6273(03)00501-4

[62] Mouravlev, A., et al. (2006) Somatic Gene Transfer of cAMP Response Element-Binding Protein Attenuates Memory Impairment in Aging Rats. Proceedings of the National Academy of Sciences of the United States of America, 103, 4705-4710. https://doi.org/10.1073/pnas.0506137103

[63] Jacobs, S., et al. (2006) Retinoic Acid Is Required Early during Adult Neurogenesis in the Dentate Gyrus. Proceedings of the National Academy of Sciences of the United States of America, 103, 3902-3907. https://doi.org/10.1073/pnas.0511294103

[64] Snyder, J.S., et al. (2005) A Role for Adult Neurogenesis in Spatial Long-Term Memory. Neuroscience, 130, 843-852.

https://doi.org/10.1016/j.neuroscience.2004.10.009

[65] von Bohlen und Halbach, O., et al. (2006) Age-Related Alterations in Hippocampal Spines and Deficiencies in Spatial Memory in Mice. Journal of Neuroscience Research, 83, 525-531. https://doi.org/10.1002/jnr.20759

[66] Jacobsen, J.S., et al. (2006) Early-onset behavioral and synaptic deficits in a mouse model of Alzheimer's disease. Proceedings of the National Academy of Sciences of the United States of America, 103, 5161-5166. https://doi.org/10.1073/pnas.0600948103

[67] Cerqueira, J.J., et al. (2007) The Prefrontal Cortex as a Key Target of the Maladaptive Response to Stress. The Journal of Neuroscience, 27, 2781-2787. https://doi.org/10.1523/JNEUROSCI.4372-06.2007

[68] Sousa, J.C., et al. (2007) Transthyretin and Alzheimer's Disease: Where in the Brain? Neurobiology of Aging, 28, 713-718. https://doi.org/10.1016/j.neurobiolaging.2006.03.015

[69] Lavado-Autric, R., et al. (2003) Early Maternal Hypothyroxinemia Alters Histogenesis and Cerebral Cortex Cytoarchitecture of the Progeny. The Journal of Clinical Investigation, 111, 1073-1082. https://doi.org/10.1172/JCI200316262

[70] Gilbert, M.E. and Sui, L. (2006) Dose-Dependent Reductions in Spatial Learning and Synaptic Function in the Dentate Gyrus of Adult Rats Following Developmental Thyroid Hormone Insufficiency. Brain Research, 1069, 10-22. https://doi.org/10.1016/j.brainres.2005.10.049

[71] Schafer, M.J., et al. (2015) Calorie Restriction Suppresses Age-Dependent Hippocampal Transcriptional Signatures. PLoS ONE, 10, e0133923. https://doi.org/10.1371/journal.pone.0133923

[72] Benoit, C.E., et al. (2011) Genomic and Proteomic Strategies to Identify Novel Targets Potentially Involved in Learning and Memory. Trends in Pharmacological 
Sciences, 32, 43-52. https://doi.org/10.1016/j.tips.2010.10.002

[73] Nunes, A.F., Saraiva, M.J. and Sousa, M.M. (2006) Transthyretin Knockouts Are a New Mouse Model for Increased Neuropeptide Y. The FASEB Journal, 20, 166-168. https://doi.org/10.1096/fj.05-4106fje

[74] Thorsell, A., et al. (2000) Behavioral Insensitivity to Restraint Stress, Absent Fear Suppression of Behavior and Impaired Spatial Learning in Transgenic Rats with Hippocampal Neuropeptide Y Overexpression. Proceedings of the National Academy of Sciences of the United States of America, 97, 12852-12857. https://doi.org/10.1073/pnas.220232997

[75] Vieira, M. and Saraiva, M.J. (2013) Transthyretin Regulates Hippocampal 14-3-3 Zeta Protein Levels. FEBS Letters, 587, 1482-1488. https://doi.org/10.1016/j.febslet.2013.03.011

[76] Schindler, C.K., Heverin, M. and Henshall, D.C. (2006) Isoform- and Subcellular Fraction-Specific Differences in Hippocampal 14-3-3 Levels Following Experimentally Evoked Seizures and in Human Temporal Lobe Epilepsy. Journal of Neurochemistry, 99, 561-569. https://doi.org/10.1111/j.1471-4159.2006.04153.x

[77] Heverin, M., et al. (2012) Proteomic Analysis of 14-3-3 Zeta Binding Proteins in the Mouse Hippocampus. International Journal of Physiology, Pathophysiology and Pharmacology, 4, 74-83.

[78] VanGuilder, H.D., et al. (2011) Hippocampal Dysregulation of Synaptic Plasticity-Associated Proteins with Age-Related Cognitive Decline. Neurobiology of Disease, 43, 201-212. https://doi.org/10.1016/j.nbd.2011.03.012

[79] Pozuelo-Rubio, M. (2011) 14-3-3Zeta Binds Class III Phosphatidylinositol-3-Kinase and Inhibits Autophagy. Autophagy, 7, 240-242.

https://doi.org/10.4161/auto.7.2.14286

[80] Pozuelo-Rubio, M. (2011) Regulation of Autophagic Activity by 14-3-3Zeta Proteins Associated with Class III Phosphatidylinositol-3-Kinase. Cell Death and Differentiation, 18, 479-492. https://doi.org/10.1038/cdd.2010.118

[81] Rajawat, Y., Hilioti, Z. and Bossis, I. (2010) Autophagy: A Target for Retinoic Acids. Autophagy, 6, 1224-1226. https://doi.org/10.4161/auto.6.8.13793

[82] Rajawat, Y., Hilioti, Z. and Bossis, I. (2011) Retinoic Acid Induces Autophagosome Maturation through Redistribution of the Cation-Independent Mannose-6-Phosphate Receptor. Antioxidants \& Redox Signaling, 14, 2165-2177. https://doi.org/10.1089/ars.2010.3491

[83] Philip, N., Acevedo, S.F. and Skoulakis, E.M. (2001) Conditional Rescue of Olfactory Learning and Memory Defects in Mutants of the 14-3-3Zeta Gene Leonardo. The Journal of Neuroscience, 21, 8417-8425.

[84] Cheah, P.S., et al. (2012) Neurodevelopmental and Neuropsychiatric Behaviour Defects Arise from 14-3-3Zeta Deficiency. Molecular Psychiatry, 17,451-466. https://doi.org/10.1038/mp.2011.158

[85] Dickson, D.W. (2004) Apoptotic Mechanisms in Alzheimer Neurofibrillary Degeneration: Cause or Effect? The Journal of Clinical Investigation, 114, 23-27. https://doi.org/10.1172/JCI22317

[86] Kang, J., et al. (1987) The Precursor of Alzheimer's Disease Amyloid A4 Protein Resembles a Cell-Surface Receptor. Nature, 325, 733-736. https://doi.org/10.1038/325733a0

[87] Philibert, K.D., et al. (2014) Identification and Characterization of ABeta Peptide Interactors in Alzheimer's Disease by Structural Approaches. Frontiers in Aging 
Neuroscience, 6, 265. https://doi.org/10.3389/fnagi.2014.00265

[88] Selkoe, D.J. (2001) Clearing the Brain's Amyloid Cobwebs. Neuron, 32, 177-180. https://doi.org/10.1016/S0896-6273(01)00475-5

[89] Cho, P.Y., et al. (2015) A Cyclic Peptide Mimic of the Beta-Amyloid Binding Domain on Transthyretin. ACS Chemical Neuroscience, 6, 778-789. https://doi.org/10.1021/cn500272a

[90] Ankarcrona, M., et al. (2016) Current and Future Treatment of Amyloid Diseases. The Journal of Internal Medicine, 280, 177-202. https://doi.org/10.1111/joim.12506

[91] Crossgrove, J.S., Li, G.J. and Zheng, W. (2005) The Choroid Plexus Removes Beta-Amyloid from Brain Cerebrospinal Fluid. Experimental Biology and Medicine (Maywood), 230, 771-776. https://doi.org/10.1177/153537020523001011

[92] Crossgrove, J.S., Smith, E.L. and Zheng, W. (2007) Macromolecules Involved in Production and Metabolism of Beta-Amyloid at the Brain Barriers. Brain Research, 1138, 187-195. https://doi.org/10.1016/j.brainres.2006.12.022

[93] Ingenbleek, Y. and Bernstein, L.H. (2015) Downsizing of Lean Body Mass Is a Key Determinant of Alzheimer's Disease. Journal of Alzheimer's Disease, 44, 745-754.

[94] Tsai, K.J., et al. (2009) Asymmetric Expression Patterns of Brain Transthyretin in Normal Mice and a Transgenic Mouse Model of Alzheimer's Disease. Neuroscience, 159, 638-646. https://doi.org/10.1016/j.neuroscience.2008.12.045

[95] Merched, A., et al. (1998) Apolipoprotein E, Transthyretin and Actin in the CSF of Alzheimer's Patients: Relation with the Senile Plaques and Cytoskeleton Biochemistry. FEBS Letters, 425, 225-228. https://doi.org/10.1016/S0014-5793(98)00234-8

[96] Stein, T.D. and Johnson, J.A. (2002) Lack of Neurodegeneration in Transgenic Mice Overexpressing Mutant Amyloid Precursor Protein Is Associated with Increased Levels of Transthyretin and the Activation of Cell Survival Pathways. Journal of Neuroscience, 22, 7380-7388.

[97] Serot, J.M., et al. (1997) Cerebrospinal Fluid Transthyretin: Aging and Late Onset Alzheimer's disease. The Journal of Neurology, Neurosurgery, and Psychiatry, 63, 506-508. https://doi.org/10.1136/jnnp.63.4.506

[98] Castano, E.M., et al. (2006) Comparative Proteomics of Cerebrospinal Fluid in Neuropathologically-Confirmed Alzheimer's Disease and Non-Demented Elderly Subjects. Neurological Research, 28, 155-163. https://doi.org/10.1179/016164106X98035

[99] Gloeckner, S.F., et al. (2008) Quantitative Analysis of Transthyretin, Tau and Amyloid-Beta in Patients with Dementia. The Journal of Alzheimer's Disease, 14, 17-25. https://doi.org/10.3233/JAD-2008-14102

[100] Hansson, S.F., et al. (2009) Reduced Levels of Amyloid-Beta-Binding Proteins in Cerebrospinal Fluid from Alzheimer's Disease Patients. The Journal of Alzheimer's Disease, 16, 389-397. https://doi.org/10.3233/JAD-2009-0966

[101] Chodobski, A. and Szmydynger-Chodobska, J. (2001) Choroid Plexus: Target for Polypeptides and Site of Their Synthesis. Microscopy Research and Technique, 52, 65-82.

https://doi.org/10.1002/1097-0029(20010101)52:1<65::AID-JEMT9>3.0.CO;2-4

[102] Geroldi, C., et al. (2000) Temporal Lobe Asymmetry in Patients with Alzheimer's Disease with Delusions. The Journal of Neurology, Neurosurgery, and Psychiatry, 69, 187-191. https://doi.org/10.1136/jnnp.69.2.187

[103] Lein, E.S., et al. (2007) Genome-Wide Atlas of Gene Expression in the Adult Mouse 
Brain. Nature, 445, 168-176. https://doi.org/10.1038/nature05453

[104] Santos, L.M., et al. (2016) Resveratrol Administration Increases Transthyretin Protein Levels Ameliorating AD Features-Importance of Transthyretin Tetrameric Stability. Molecular Medicine, 22, 28. https://doi.org/10.2119/molmed.2016.00124

[105] Maetani, Y., et al. (2016) Familial Amyloid Polyneuropathy Involving a Homozygous Val30Met Mutation in the Amyloidogenic Transthyretin Gene Presenting with Superficial Siderosis: A Case Report. Rinsho Shinkeigaku, 56, 430-434.

[106] Li, X. and Buxbaum, J.N. (2011) Transthyretin and the Brain Re-Visited: Is Neuronal Synthesis of Transthyretin Protective in Alzheimer's Disease? Molecular Neurodegeneration, 6, 79. https://doi.org/10.1186/1750-1326-6-79

[107] Dewachter, I., et al. (2000) Aging Increased Amyloid Peptide and Caused Amyloid Plaques in Brain of Old APP/V717I Transgenic Mice by a Different Mechanism than Mutant Presenilin1. Journal of Neuroscience, 20, 6452-6458.

[108] Natunen, T., et al. (2012) Genetic Analysis of Genes Involved in Amyloid-Beta Degradation and Clearance in Alzheimer's Disease. The Journal of Alzheimer's Disease, 28, 553-559.

[109] Bergen, A.A., et al. (2015) Gene Expression and Functional Annotation of Human Choroid Plexus Epithelium Failure in Alzheimer's Disease. BMC Genomics, 16, 956. https://doi.org/10.1186/s12864-015-2159-Z

[110] Bastianetto, S., Brouillette, J. and Quirion, R. (2007) Neuroprotective Effects of Natural Products: Interaction with Intracellular Kinases, Amyloid Peptides and a Possible Role for Transthyretin. Neurochemical Research, 32, 1720-1725.

https://doi.org/10.1007/s11064-007-9333-x 